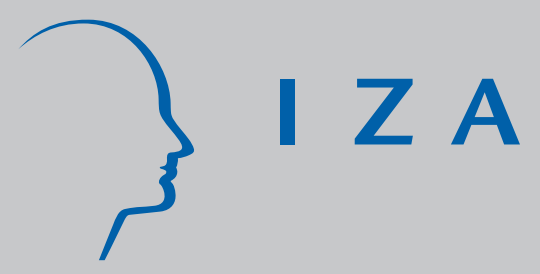

IZA DP No. 1423

On the Notion of Responsibility in Organizations

Dirk Sliwka

December 2004 


\title{
On the Notion of Responsibility in Organizations
}

\author{
Dirk Sliwka \\ University of Cologne \\ and IZA Bonn
}

\section{Discussion Paper No. 1423 \\ December 2004}

\author{
IZA \\ P.O. Box 7240 \\ 53072 Bonn \\ Germany \\ Phone: +49-228-3894-0 \\ Fax: +49-228-3894-180 \\ Email: iza@iza.org
}

\begin{abstract}
Any opinions expressed here are those of the author(s) and not those of the institute. Research disseminated by IZA may include views on policy, but the institute itself takes no institutional policy positions.

The Institute for the Study of Labor (IZA) in Bonn is a local and virtual international research center and a place of communication between science, politics and business. IZA is an independent nonprofit company supported by Deutsche Post World Net. The center is associated with the University of Bonn and offers a stimulating research environment through its research networks, research support, and visitors and doctoral programs. IZA engages in (i) original and internationally competitive research in all fields of labor economics, (ii) development of policy concepts, and (iii) dissemination of research results and concepts to the interested public.
\end{abstract}

IZA Discussion Papers often represent preliminary work and are circulated to encourage discussion. Citation of such a paper should account for its provisional character. A revised version may be available directly from the author. 
IZA Discussion Paper No. 1423

December 2004

\section{ABSTRACT}

\section{On the Notion of Responsibility in Organizations}

We derive a natural definition of responsibility in a formal model where employees care for their career prospects: A superior holds a subordinate responsible for a task, when she announces her beliefs that this subordinate contributes most to this task. We show, that those announced beliefs lead to a self fulfilling prophecy as the reputation of the responsible subordinate then is affected by the outcome of the task and he therefore has strong incentives to contribute to its success. There are equilibria where either a single agent or no agent is responsible for a task but joint responsibility never arises. Several extensions are discussed.

JEL Classification: D23, M12

Keywords: responsibility, career concern, reputation, delegation, incentives

Dirk Sliwka

University of Cologne

Herbert-Lewin-Str. 2

50937 Cologne

Germany

Email: dirk.sliwka@uni-koeln.de 
"The sole and undivided responsibility of one man will naturally beget a livelier sense of duty and a more exact regard to reputation."

Alexander Hamilton

\section{Introduction}

When a certain task has to be carried out in an organization several people may contribute to the success, but typically a superior will hold one person responsible for the task. But what is the exact content of the expression "holding someone responsible"? And why is it considered to be necessary to hold someone responsible in so many instances? In this paper a simple theory is provided of what responsibility means in a multi-agent career concerns framework.

To illustrate the key idea consider a situation in which a manager of a certain department delegates a certain task or project to some of her subordinates. After having observed the outcome of the project, she will most certainly adapt her opinion on the talent of all individuals who have contributed to the task. If now this manager believes that the largest part of the work has been carried out by one employee, and the project has been a success, she will adapt her opinion on this employee's talent to a larger extent than her opinion on other employees. But in many instances a high opinion by a superior will pay off in the future. Due to those considerations, the employee who is believed to be contributing most, may then have indeed a higher incentive to contribute more than his colleagues. If the superior is not able to monitor the individual contributions to the task, her beliefs thus may become self-fulfilling. The larger she believes one agent's contribution to be, the stronger his reputation will be affected by the outcome as "he will be held responsible" to a larger extent for the success of task. But the stronger his reputation is affected, the stronger will be his incentives to work hard on the task.

When several agents can possibly contribute to the task, there will of course be a multiplicity of equilibria as the extent of each agent's contribu- 
tion is essentially affected in equilibrium by the beliefs of the superior on the respective contributions. This leads to a natural notion of responsibility: A superior holds a certain subordinate responsible for a task, when she announces her beliefs that this subordinate contributes most to this task. But, the best response of this subordinate will then be indeed to contribute more to the task than others. Hence, from this perspective the informal allocation of responsibility in organization, which typically comes along without any change in formal contracts, can be interpreted precisely as the selection of one of several equilibria.

Other authors in economics have explored different notions of responsibility. In Prendergast (1995) the responsibility of an agent is defined as the subset of tasks allocated to him by a manager and it is shown that rent seeking considerations lead the manager to allocate to few tasks to the agent. Manove (1997) defines job responsibility as the extent to which an agent's effort affects the principal's return and examines how wages and promotion policies are related to job responsibility.

The incentive effects of career concerns have been first formally analyzed by Holmström (1982). In his basic framework an agent's output is the sum of his effort, his normally distributed ability which is initially unknown to all and some random component. This output is observed by potential employers on a competitive labor market who bid for the agent's services. In the unique equilibrium an agent works hard in the beginning of his career to affect the market's beliefs on his ability and receive higher wage offers. Dewatripont et al. (1999b) generalize Holmström's model including the possibility that an agent's talent also determines his marginal product of effort. ${ }^{1}$ In such a framework a multiplicity of equilibria arises in a single agent model.

We adopt the idea that an agent's imperfectly known talent determines his marginal product of effort. But whereas in the career concerns literature incentives are typically created by a competitive labor market paying an agent a wage proportional to his expected ability, in our model incentives arise due to an internal promotion decision such as for instance in the tournament literature (compare Lazear and Rosen (1981), Nalebuff and Stiglitz (1983) or Green and Stokey (1983)) or more specific in the literature on pro- 
motions as for instance in Waldman (1990), Prendergast (1993) or Fairburn and Malcomson (1994) and (2001). This seems a more natural assumption for modelling career concerns within an organization, as no perfect visibility of output to the outside labor market is necessary. In our model, two agents with imperfectly known abilities contribute effort to a certain task. A principal observes the outcome of the task and can then promote one of the two agents. In contrast to the tournament literature, however, the principal cannot observe individual signals on the performance of each agent separately, but only learns the total outcome to which both agents can possibly contribute.

We will show that there are two types of equilibria in our model. On the one hand, under certain restrictions two sole-responsibility-equilibria exist in which exactly one of the two agents exerts a positive effort level and is held responsible for the task. On the other hand, there is always a noresponsibility-equilibrium in which no agent contributes effort but there is never an equilibrium where both agents contribute positive effort levels. This result formalizes the idea that in organizations it is commonly felt that one should assign sole responsibility for a task to a unique person as has already been emphasized in the quotation above.

Note that the choice of responsibility does not encompass the allocation of a formal decision right. The principal is not able to commit ex-ante on the consequences of the outcome as this seems to be an important feature of most decision procedures within organizations: ${ }^{2}$ When a superior announces to some employee "You are responsible for this task" this is rather an informal act. No contract is signed which is enforced by third parties. In the model we try to capture this notion of responsibility.

In this view the precise economic meaning of this sentence is that the principal announces that she believes in a large contribution of the specific agent to the particular task and therefore will promote this agent afterwards if the task is succesfull. If an announcement is consistent with one of the possible equilibrium strategies, the principal will indeed have an incentive to do what she announced given that the agents believe its content. In the terminology of Farrell and Rabin (1996) in their survey on cheap talk, 
the principal's messages is therefore self-comitting: "if believed, it creates incentives for the speaker to fulfill it" (p. 111). When the agents optimally react to this announcement of beliefs it will become self-fulfiling without any commitment. Hence, the informal act of allocating responsibility does have economic consequences.

If the principal can select one of the possible equilibria, we have to ask which one she prefers. It is straightforward to see that this will never be the no-responsibility-equilibrium. Hence, in a next step, we compare the two sole responsibility equilibria if the agents differ in the principal's prior expectations on their respective abilities. As we will show, there are two aspects that should be considered when determining who should be held responsible for a certain task. On the one hand, we examine the incentive aspect of responsibility and characterize which agent works harder when being responsible and which one produces a higher expected output. But a second aspect is that by allocating responsibility, the principal can learn more about the talent of the responsible agent and this will pay off in the future. Hence, we also analyze who should be held responsible when this learning aspect is considered. ${ }^{3}$

In the single task model, however, an inefficiency arises as at least one of the agents does never exerts any effort on the task. Therefore we extend the model to encompass a second task. We show that in the extended model three types of equilibria occur: Again, there is a no-responsibility equilibrium in which neither of the agents exerts any effort. Second, there is a continuum of sole responsibility equilibria, in which a single agent works for both tasks. But finally, there are equilibria in which each agent is responsible for a different task. In this equilibrium both agents compete in a tournament structure each one by exerting effort on the task for which he is held responsible. We show that with this alloaction of responsibility on separate tasks, the principal may well be able to achieve first-best incentives when he can adapt the agents' benefit from promotion.

Similar to the single task model there will never be equilibria in which the responsibility for one task is divided among the two agents. Again incentives can only created with the "sole and undivided responsibility of one man" for one task. 
The paper proceeds as follows. In section 2 we describe the simple model. In the subsequent section 3 we analyze how the promotion decision is made by the principal. After this, the possible equilibria of the game are characterized in section 4 . Sections 5 and 6 consider the incentive and the learning aspect of responsibility. Finally in section 7 an extension of the model on multiple tasks is studied.

\section{The Model}

We consider the following simple model. A principal employs two agents $i=1,2$. Each agent has an initially unknown ability $\theta_{i}$. The principal and the agents share a common prior belief on each agent's ability and the abilities independently normally distributed

$$
\theta_{i} \sim N\left(m_{i}, \sigma_{i}^{2}\right) \text { for } i=1,2
$$

with both $m_{i}>0$. Hence, initially principal and agents are symmetrically informed. In a first period both agents can exert effort $a_{i}$ on a task. They have an identical convex cost function $c(a)$, with

$$
c(0)=0 \text { and } c^{\prime}(0)=0 \text {. }
$$

The output of the task accrues to the principal and is given by the sum of the individual contributions

$$
y=\theta_{1} a_{1}+\theta_{2} a_{2} .
$$

Hence, the ability of an agent determines the marginal productivity of his effort. The higher his ability, the more productive is a given amount of effort exerted by an agent. The principal only observes the total output and not the individual contributions.

In the second period one of the two agents gets promoted to a better job by the principal. The principal cares for having the better agent promoted but she cannot commit to a promotion policy in advance. For simplicity we assume that the principal's payoff from the promotion decision is a linear 
function of the ability of the agent who is promoted, i.e. in expected terms her second period output is $E\left[R \cdot \theta_{i} \mid y\right]$ where $R$ is a given constant. The agent who is promoted receives a benefit $B$ from promotion, which may simply be the wage differential between the old and the new job. In most of the paper we treat $B$ as exogenously given and do not consider endogenous wage setting. As for instance Waldman (1984) and in a tournament framework Zabojník and Bernhardt (2001) have laid out, wage increases due to promotions are to a large extent beyond a firm's control as a promotion signals a high worker productivity to other employers whose external wage offers have the to be matched by the current employer. ${ }^{4}$ We further discuss the endogenization of $B$ in the last section.

\section{The Promotion Decision}

We look for pure strategy perfect Bayesian equilibria of the model described above. First, the optimal promotion decision in period 2 is analyzed as the principal's reaction function given the principal's beliefs on the agents' equilibrium effort levels dentoted by $a_{1}$ and $a_{2}$. In the next section we will then derive the agent's equilibrium effort choices given this promotion strategy. Of course, the principal wants to promote the agent with the higher expected ability, i.e. agent $i$ is always promoted if

$$
E\left[\theta_{i} \mid y\right]>E\left[\theta_{j} \mid y\right]
$$

after the principal has observed the actual output $y$. The principal is indifferent, whom to promote when $E\left[\theta_{i} \mid y\right]=E\left[\theta_{j} \mid y\right]$. Due to the normality assumption the expected ability of agent $i$ is given by ${ }^{5}$

$$
E\left[\theta_{i} \mid y\right]=m_{i}+\frac{a_{i} \sigma_{i}^{2}}{a_{i}^{2} \sigma_{i}^{2}+a_{j}^{2} \sigma_{j}^{2}}(y-E[y]) .
$$

for strategies $a_{1}$ and $a_{2}$ chosen by the agents. Note that although she cannot observe the effort levels, the principal infers $a_{1}$ and $a_{2}$ in a pure strategy equilibrium. The principal adapts her beliefs on the ability of each agent after she has observed the outcome of the task $y$ by taking into account the 
equilibrium strategies played by the agents in the first period. If the task is completed successfully and the outcome is higher than the expected outcome given the principal's beliefs on the agents' contributions (i.e. $y>E[y]$ ), the reputation of both agents raises above the prior level, otherwise it decreases.

The strength of the adaptation depends on the respective effort contributions of both agents and the precision of the knowledge on their abilities. Comparing both agents, note that if $a_{i} \sigma_{i}^{2}>a_{j} \sigma_{j}^{2}$ then the beliefs on the ability of agent $i$ are adapted to a stronger extent than those on agent $j$. In particular, if the initial beliefs on the abilities are identical the adaptation is stronger for the agent with the larger presumed contribution.

Applying those considerations we can derive the principal's optimal second period promotion decision given the agent's equilibrium actions.

Lemma 1 Suppose that in equilibrium $\sigma_{i}^{2} a_{i}>\sigma_{j}^{2} a_{j}$. In that case agent $i$ is promoted if the realized profit exceeds a certain performance standard $y_{T}$, which is given by

$$
y_{T}=E[y]-\frac{a_{i}^{2} \sigma_{i}^{2}+a_{j}^{2} \sigma_{j}^{2}}{a_{i} \sigma_{i}^{2}-a_{j} \sigma_{j}^{2}}\left(m_{i}-m_{j}\right) .
$$

Furthermore, if the initial beliefs on the agent's abilities are identical, agent $i$ will be promoted if profits are larger than the initially expected profits.

If however, in equilibrium $\sigma_{i}^{2} a_{i}=\sigma_{j}^{2} a_{j}$ the principal will always promote the agent with the higher initially expected ability.

Proof: See Appendix.

If initial beliefs on both agents are identical - for instance because both are new to the organization - the agent with the higher equilibrium effort is promoted if and only if profits are higher than expected profits. As we have seen above, both agents' reputation is always adapted in the same direction if they both contribute positive effort levels. A higher than expected outcome increases the principal's beliefs on both agents' abilities, a lower than expected outcomes reduces those beliefs. But in addition, the adaptation of beliefs is stronger for the agent with the higher presumed contribution. 
Hence, it is him who gets promoted if the task is a success $(y>E[y])$ and his colleague in case of a failure $(y<E[y])$.

If, however, agent $i$ is initially believed to be more able than his colleague (i.e. $m_{i}>m_{j}$ ), his profit target is lower than the initially expected profit. It takes a comparatively worse result to edge him out and this "discount" is larger, the larger the initial ability difference.

\section{Responsibility and Incentives}

Given the optimal promotion decision by the principal, we can now analyze the equilibrium effort levels exerted by the agents. The agent's decisions must be best responses given the principal's beliefs on the effort contributions by the agents and her promotion strategy in period 2. Now it is important to distinguish between actual effort levels and the principal's beliefs. We denote those beliefs on the agent's actions by $\hat{a}_{i}$ and $\hat{a}_{j}$ and the expected output for given beliefs by $\hat{y}$. It is important to note that the principal makes his promotion decision based on these beliefs. Hence, the threshold for promotion from the agent's view in the first period will be based on this beliefs:

$$
y_{T}=E[\hat{y}]-\frac{\hat{a}_{i}^{2} \sigma_{i}^{2}+\hat{a}_{j}^{2} \sigma_{j}^{2}}{\hat{a}_{i} \sigma_{i}^{2}-\hat{a}_{j} \sigma_{j}^{2}}\left(m_{i}-m_{j}\right) .
$$

The agents anticipate that agent $i$ will be promoted when the actual output $y=\theta_{i} a_{i}+\theta_{j} a_{j}$ exceeds this threshold. Although the beliefs will of course be correct in equilibrium, we have to consider the agents' possible deviations.

First, suppose that the principal's beliefs are such that for one agent $i$ condition $\hat{a}_{i} \sigma_{i}^{2}>\hat{a}_{j} \sigma_{j}^{2}$ holds. Agent $j$ will then be promoted only if profits are smaller than a certain cut-off value. Hence, the best he can do is to exert no effort at all. A good outcome of the task will always increase his colleague's reputation to a larger extent than his own. Hence, it is straightforward that in any equilibrium with $\hat{a}_{i} \sigma_{i}^{2}>\hat{a}_{j} \sigma_{j}^{2}$ we must have that $a_{j}=0$.

In that case, agent $i$ bears sole responsibility for the task. It is only him, who contributes to the task. Hence, only the beliefs on his talent are adapted according to the outcome of the task. According to Lemma 1, he will then 
be promoted if the outcome of the task exceeds

$$
y_{T}=E[\hat{y}]-\hat{a}_{i}\left(m_{i}-m_{j}\right)=\hat{a}_{i} m_{j} .
$$

Hence, the agent has to beat a performance standard, determined by the product of his own conjectured contribution and his colleague's expected ability. To understand the value of the standard, just think of the principal asking the following hypothetical question: "Given my initial beliefs, which profit would agent $j$ attain in expected terms if he exerted the same effort level that I think agent $i$ has exerted?". If the profit generated by $i$ exceeds this value, then agent $i$ will be promoted otherwise $j$ is preferred.

In period 1 agent $i$ then maximizes the following expression, given the principal's equilibrium beliefs:

$$
\max _{a_{i}} \operatorname{Pr}\left(\theta_{i} a_{i}>\hat{a}_{i} m_{j}\right) B-c\left(a_{i}\right) .
$$

If there is a unique solution to this problem with a positive $a_{i}=\hat{a}_{i}$ we have an equilibrium. Indeed, if the uncertainty on agent $i$ 's ability is not too small, such an equilibrium exists as is shown in the following result: ${ }^{6}$

Proposition 1 For each agent $i$, if $\sigma_{i}$ is sufficiently large there exist a "sole responsibility" equilibrium, in which agent $i$ contributes a strictly positive effort level $a_{i}^{*}$, determined by

$$
\frac{m_{j} B}{\sigma_{i}} \phi\left(\frac{m_{j}-m_{i}}{\sigma_{i}}\right)=c^{\prime}\left(a_{i}^{*}\right) a_{i}^{*} .
$$

and the other agent supplies no effort at all $\left(a_{j}^{*}=0\right)$. The principal will promote agent $i$ if the outcome exceeds the expected outcome when $j$ instead of $i$ exerted effort level $a_{i}^{*}$, that is, $i$ is promoted if

$$
y \geq a_{i}^{*} m_{j} .
$$

Proof: See Appendix.

To understand why we can show the existence of such an equilibrium only if the variance of the agent's ability is large just note that if it is zero 
the principal knows the agent's abilities perfectly and the outcome of the task will not affect her beliefs. There must be some uncertainty on the agent's abilities such that they indeed have an interest to demonstrate their respective abilities by exerting a high effort level.

It is interesting to note that if we would allow for sabotage as for instance in the tournament model by Lazear (1989), then the agent $j$ would have an incentive to use this possibility and destroy the output produced by agent $i$ as this raises his own career prospects.

Now suppose that we have an equilibrium where $\hat{a}_{i} \sigma_{i}^{2}=\hat{a}_{j} \sigma_{j}^{2}$. In that case the principal cannot learn from the realized profit as we know from Lemma 1. If in that case the initially expected abilities differ, the agent with the higher expected ability will always be promoted. But then neither the more, nor the less able agent has any incentive to exert effort. Hence, if expected abilities differ we must have that $a_{i}=a_{j}=0$ whenever $a_{i} \sigma_{i}^{2}=a_{j} \sigma_{j}^{2}$ and no agent exerts effort in equilibrium. If the initially expected abilities coincide the principal is indifferent between promoting each of the agents. Hence, she may apply an arbitrary promotion strategy in this case. When the promotion strategy does not condition on the observed output, we again obtain an equilibrium in which indeed no agent exerts effort. But in addition, we show in the subsequent result, that even with an arbitrary promotion strategy conditional on output there is no equilibrium, in which both agents exert positive effort levels:

Proposition 2 There is always a "no responsibility" equilibrium in which no agent exerts any effort. If the expected abilities of the agents differ, the agent with the higher expected ability is always promoted. Furthermore, there is no equilibrium in which both agents exert positive effort levels.

Proof: See Appendix.

Hence, there is either a situation in which exactly one of the agents is responsible for the task or a situation without any responsibility. But in the latter case no agent contributes to the success of the task, as no one can gain anything relative to his colleague in the promotion tournament. 
From now on we assume that both $\sigma_{i}$ and $\sigma_{j}$ are sufficiently large such that the two sole responsibility equilibria indeed exist as we want to compare the two possible equilibria. ${ }^{7}$ Furthermore, we suppose that the principal can choose among the mentioned equilibria by holding one of the agents responsible for the task. It is implicit in this assumption that the players can coordinate on one equilibrium by some form of cheap talk by the principal. The principal can make an announcement like "I hold agent $i$ responsible for the task" before the game starts which corresponds in our model to an announcement "I will promote agent $i$ if the output is larger than $e_{i}^{*} m_{j}$ ".

Note that if an announcement is consistent with one of the possible equilibrium promotion strategies, the principal will indeed have an incentive to do what she announced given that the agents believe its content: By definition of the equilibrium, in these three cases the principal will optimally follow the announcement given that the agent's play a best response to the announced strategy. As laid out in the introduction, each of those messages is therefore self-comitting in the sense of Farrell and Rabin (1996).

For our purpose, it seems appropriate to check which equilibrium (and therefore which of the self-committing announcements) generates the highest profits for the principal. It is straightforward, that this cannot be the no responsibility equilibrium. Hence, we will examine which of the two agents should be held responsible for the task.

\section{Who works harder?}

To answer this question we have to compare the two sole responsibility equilibria. First, we suppose that both agents have different prior expected ability levels, but that the information on both agents is equally precise (i.e. $\left.\sigma_{i}^{2}=\sigma_{j}^{2}\right)$. Without loss of generality we assume that $i$ is the agent with the higher expected ability, i.e. $m_{i}>m_{j}$. The difference in abilities is denoted by $\Delta m=m_{i}-m_{j}$.

As $\phi($.$) is symmetric it can be seen from equation (3) that for a given$ difference in abilities $\Delta m$ the effort of the responsible agent is the higher the 
higher his colleague's ability. Hence, the agent with the lower ability works harder if he is held responsible than his colleague with a higher expected ability in the same situation! However, it is less clear how the total output is affected as the more able agent has a higher marginal product of output. This is examined in the following Proposition:

Proposition 3 Suppose that the information on both agents' abilities is equally precise. Then the agent with the lower expected ability contributes more effort when he is responsible than the agent with the higher expected ability. However, the total expected outcome is higher when the more able agent is responsible.

Proof: See Appendix.

To understand the result that the agent with the lower expected ability will supply a higher effort level, note that the optimal promotion rule by the principal yields a threshold value for the generated profits such that the responsible agent is promoted if and only if profits are higher than this value. But the threshold value is determined by the expected ability of the agent who is not responsible. For the more able agent it is easier to attain this value than for the less able agent for two reasons: first, the threshold is lower and in addition his marginal product of effort is higher such that it easier for him to attain the threshold with a sufficiently high probability.

Still, the more able agent produces a higher expected total output with a given effort level as he has a higher marginal product of effort. As the result shows, for this reason the total output of the more able agent will be higher although he exerts less effort. Hence, for equal precisions the principal prefers that the more able agent is held responsible and works for the task.

After having considered the case where the expectations on the agents' abilities differ but are equally precise, it seems interesting to examine the polar case, where the expected abilities coincide, but the precisions differ. For $\Delta m=0$ equation (3) yields

$$
\frac{m_{j} B}{\sigma_{i}} \phi(0)=c^{\prime}\left(a_{i}^{*}\right) a_{i}^{*}
$$


The left hand side is decreasing in the variance. Hence, it can directly be seen that the agent with the more precise signal will exert a higher equilibrium effort if he is held responsible for the task. As the expected marginal product is the same for both agents this directly implies that the expected output must be higher if this agent is held responsible.

To understand this observation note that the marginal product of effort in equilibrium (at $a_{i}=\hat{a}_{i}$ ) is larger for an agent with a smaller variance: To see that, consider equation (2) and note that for $m_{i}=m_{j}$ the marginal product of effort is determined by the density of a normal distribution at its mean at $a_{i}=\hat{a}_{i}$. But the density at the mean is higher the lower the variance. On a less technical level, when less is known on the agent's ability and therefore on his marginal product of effort, there is a higher variance of the possible outcome for a given effort level. Hence, the impact of effort on the outcome of the tournament is weak relative to the impact of chance. Therefore exerting effort is less attractive for an agent who is less sure on his ability than for an agent who knows his productivity more precisely. This result is related to a typical result from the tournament literature where for a given prize spread the equilibrium effort is higher the lower the variance of the noise terms.

We can summarize those considerations in the following result:

Proposition 4 If both agents have the same expected ability but differ in the precision with which the respective abilities are known, the agent with the more precisely known ability exerts more effort and produces a higher output.

So far we have only considered the impact of responsibility on the current output. But contrary to typical career concerns models where future returns accrue to the agent due to a perfectly competitive labor market, in our model the firm also cares for promoting the better agent. As we will see in the next section this learning purpose is also important to figure out who should best be held responsible for carrying out the task. 


\section{How to learn most?}

If an agent's career goes on within the same organization, it will of course be important to promote the agent with the higher expected ability. Recall the assumption, that when promoting an agent with expected ability $E\left[\theta_{i} \mid y\right]$ the principal receives a payoff of $E\left[R \cdot \theta_{i} \mid y\right]$. First, we consider a situation where the principal only cares for this second period payoff to isolate the learning effects from the incentive effects of allocation responsibility.

Note that the firm learns nothing on the agent who is not responsible. Hence, the interesting question is from which agent the firm can learn more to make an appropriate promotion decision. Suppose that agent $i$ was responsible for carrying out the task and a profit of $y_{i}$ has been realized. As we have seen, the firm will promote agent $i$ if $E\left[\theta_{i} \mid y_{i}\right]>m_{j}$. In that case, the firm's return on the second stage is $R \cdot \max \left\{E\left[\theta_{i} \mid y_{i}\right], m_{j}\right\}$. The prior expectation of the firm's profits on the second stage when holding agent $i$ responsible for the task are therefore given by

$$
R \cdot E\left[\max \left\{E\left[\theta_{i} \mid y_{i}\right], m_{j}\right\}\right]
$$

This can be reformulated using equation (1), the expressions for $y$ and $E[y]$ and the fact that $a_{j}=0$ if agent $i$ is held responsible for carrying out the task:

$$
R \cdot E\left[\max \left\{m_{i}+\frac{1}{\hat{a}_{i}}(y-E[y]), m_{j}\right\}\right]=R \cdot E\left[\max \left\{\theta_{i}, m_{j}\right\}\right]
$$

If the firm only cares for second period profits, she will assign responsibility to agent $i$ instead of agent $j$ if

$$
E\left[\max \left\{\theta_{i}, m_{j}\right\}\right] \geq E\left[\max \left\{\theta_{j}, m_{i}\right\}\right] .
$$

By computing those two expressions and comparing them, we obtain the following clear-cut result:

Proposition 5 If the principal only cares to promote the better agent, she should allocate responsibility to the agent whose ability is less certain regardless of the agents' expected abilities. 
Proof: See Appendix.

Hence, if she only cares for learning about the agents' abilities the principal should hold the agent responsible on whose ability less is known, whatever the initially expected abilities of both. It is this agent, on which there is more to learn. ${ }^{8}$ To understand this result suppose that agent $i$ was held responsible for the task. Note that after having observed the outcome the principal can always promote agent $j$ and receive an expected return of $R \cdot m_{j}$. She will promote agent $i$ only if his posterior expected ability exceeds $m_{j}$. If the uncertainty on $i$ 's ability is higher, so will be the prior variance of $E\left[\theta_{i} \mid y_{i}\right]$. But then the "option value" of such an agent is higher for the principal as there is more weight in the upper tail of the distribution.

In a certain sense, it is beneficial to assign responsibility to the less precisely known agent simply as there is more to learn on him than on his colleague. Note that this result holds whatever the prior expected abilities are.

Now consider the case where both agents have equal prior expected abilities but differ in the precision with which those abilities are known. Note that in this case the result of Proposition 5 contrasts that of Proposition 4 as when considering the incentive aspect the agent with the more precisely known ability should be promoted, but when only taking the learning aspect into account the contrary is true. A direct implication is the following result:

Corollary 1 If the agents' expected abilities are identical, the principal should assign responsibility to the agent with the more precisely known ability if the importance of the future task $R$ is sufficiently small, otherwise she should assign responsibility to the agent with the less precisely known ability.

Hence, if it is sufficiently important to promote the better qualified agent, then the principal should assign responsibility to the agent whose ability is less well known. 


\section{Multiple Tasks}

Note that in the model of the previous section, in any equilibrium at least one of the agents is always idle. This is clearly inefficient as the marginal costs of effort are zero for this agent when exerting more effort. However, if there will be more tasks, we can show that this inefficiency disappears.

We therefore extend the model to encompass the case of multiple tasks. In the first period each agent $i$ can now exert efforts $a_{i A}$ and $a_{i B}$ on two separate tasks $A$ and $B$. Both agents have an identical convex cost function $c\left(a_{i A}+a_{i B}\right)$. Note that we assume that an agent's efforts for the two tasks are perefctly substitutable in his cost function. For simplicity we consider a symmetric situation and therefore assume that the prior expectations on the agents' abilities are identical and, hence,

$$
\theta_{i} \sim N\left(m, \sigma^{2}\right) \text { for } i=1,2
$$

The outcome of task $t$ is now given by

$$
y_{t}=\theta_{1} a_{1 t}+\theta_{2} a_{2 t} .
$$

Hence, if both agents exert effort for both tasks, the principal has two signals on the talent of each agent. As before, she will promote the agent with the higher expected ability, i.e. agent $i$ is always promoted if $E\left[\theta_{i} \mid y_{A}, y_{B}\right]>$ $E\left[\theta_{j} \mid y_{A}, y_{B}\right]$. For ease of notation define

$$
\theta=\left(\begin{array}{c}
\theta_{1} \\
\theta_{2}
\end{array}\right), y=\left(\begin{array}{l}
y_{A} \\
y_{B}
\end{array}\right), \text { and } \mathbf{A}=\left(\begin{array}{cc}
a_{1 A} & a_{2 A} \\
a_{1 B} & a_{2 B}
\end{array}\right) .
$$

Hence, the vector of outputs $y=\mathbf{A} \theta$. We can now compute the conditional expectation of $\theta$ given the observed outputs $y$. The covariance matrix of $y$ is given by $\operatorname{Var}[y]=\mathbf{A} \operatorname{Var}[\theta] \mathbf{A}^{\prime}$. We now have to distinguish two cases, depending on whether $\operatorname{Var}[y]$ is invertible for given equilibrium effort levels, which is the case if and only if $\mathbf{A}$ is nonsingular or $a_{i A} a_{j B}-a_{j A} a_{i B} \neq 0$.

In the first case $a_{i A} a_{j B}=a_{j A} a_{i B}$ such that the effort vectors are linear dependent. Then either $y_{A}$ and $y_{B}$ contain the same information or at most one of them contains information on $\theta_{1}$ and $\theta_{2}$. When furthermore $a_{i A}=a_{j A}$ 
and $a_{j B}=a_{j B}$ then the principal cannot learn anything from the outcome. By the same logic as in Section 4 the only feasible equilibrium in this case will be a no-responsibility equilibrium with $a_{i A}=a_{j B}=a_{j A}=a_{i B}=0$. When only $a_{i A}>0$ and $a_{j A}=a_{i B}=a_{j B}=0$ then the principal cannot learn anything on $B$ 's ability and $A$ works only on a single task. We are then back in the case of the previous section and we can again find an equilibrium characterized by equation $3 .^{9}$

When neither of this is the case we can proceed with equation (1) and obtain

$E\left[\theta_{i} \mid \theta_{i} a_{i A}+\theta_{j} a_{j A}\right]=m+\frac{a_{i A}}{a_{i A}^{2}+a_{j A}^{2}}\left(y_{A}-E\left[y_{A}\right]\right)=m+\frac{a_{i B}}{a_{i B}^{2}+a_{j B}^{2}}\left(y_{B}-E\left[y_{B}\right]\right)$.

Hence, $i$ is promoted whenever

$$
\begin{gathered}
\frac{a_{i A}}{a_{i A}^{2}+a_{j A}^{2}}\left(y_{A}-E\left[y_{A}\right]\right)>\frac{a_{j A}}{a_{i A}^{2}+a_{j A}^{2}}\left(y_{A}-E\left[y_{A}\right]\right) \Leftrightarrow \\
\left(a_{i A}-a_{j A}\right)\left(y_{A}-E\left[y_{A}\right]\right)>0 .
\end{gathered}
$$

When $a_{i A}>a_{j A}$ then agent $i$ is promoted whenever $y_{A}>E\left[y_{A}\right]$. Hence, at stage 1 we will always have that $a_{j A}=0$ in this case. But note that as $a_{i A} a_{j B}=a_{j A} a_{i B}$, this implies that $a_{i B}>a_{j B}$ and thus $a_{j B}=0$. Hence, if $a_{i A} a_{j B}=a_{j A} a_{i B}$ it can only be the case that one of both agents works for both tasks whereas the other one does not exert effort for either of the tasks. Indeed, we can show that such equilibria exist:

Proposition 6 There is a continuum of equilibria in which only one of the agents is responsible for both tasks. The responsible agent $i$ will choose $a_{i A}^{*}$ and $a_{i B}^{*}$ such that $a_{i A}^{*}+a_{i B}^{*}=a_{i}^{*}$ where $a_{i}^{*}$ is defined by

$$
\phi(0) \frac{m}{\sigma_{i}} B=c^{\prime}\left(a_{i}^{*}\right) a_{i}^{*} .
$$

These equilibria exist if $\sigma_{i}$ is sufficiently large. Furthermore, there is always $a$ "no responsibility" equilibrium in which no agent exerts effort on any of the tasks.

Proof: See Appendix. 
Note that the effort exerted by the responsible agent on both tasks exactly coincides with the effort exerted in a sole responsibility equilibrium in the single task model. Hence, in the equilibria characterized so far the total effort exerted will never exceed that in the single task model.

In the second case $a_{A i} a_{B j}-a_{A j} a_{B i} \neq 0$ and the matrix $A$ is nonsingular. Now we can directly compute ${ }^{10}$

$$
E[\theta \mid y]=E[\theta]+\operatorname{Cov}[\theta, y](\operatorname{Var}[y])^{-1}(y-E[y]) .
$$

Using that $\operatorname{Cov}[\theta, y]=\operatorname{Cov}[\theta, \mathbf{A} \theta]=\operatorname{Var}[\theta] \mathbf{A}^{\prime}$ we obtain that

$$
\begin{aligned}
E[\theta \mid y] & =E[\theta]+\operatorname{Var}[\theta] \mathbf{A}^{\prime}\left(\mathbf{A} \operatorname{Var}[\theta] \mathbf{A}^{\prime}\right)^{-1}(y-E[y]) \\
& =E[\theta]+\mathbf{A}^{-1}(y-E[y]) .
\end{aligned}
$$

From this expression we can derive the principal's beliefs on the agents's respective abilities after he has observed the outcome of both tasks

$$
E\left[\theta_{i} \mid y\right]=m+\frac{a_{j B}\left(y_{A}-E\left[y_{A}\right]\right)-a_{j A}\left(y_{B}-E\left[y_{B}\right]\right)}{a_{i A} a_{j B}-a_{j A} a_{i B}} .
$$

Hence, agent $i$ is promoted whenever

$$
\frac{a_{j B}\left(y_{A}-E\left[y_{A}\right]\right)-a_{j A}\left(y_{B}-E\left[y_{B}\right]\right)}{a_{i A} a_{j B}-a_{j A} a_{i B}}>\frac{a_{i B}\left(y_{A}-E\left[y_{A}\right]\right)-a_{i A}\left(y_{B}-E\left[y_{B}\right]\right)}{a_{j A} a_{i B}-a_{i A} a_{j B}}
$$

Suppose now w.l.o.g. that $a_{i A} a_{j B}>a_{j A} a_{i B}$. Note that when all efforts are positive, this is equivalent to $\frac{a_{i A}}{a_{i A}+a_{j A}}>\frac{a_{i B}}{a_{i B}+a_{j B}}$. Hence, agent $i$ spends a higher fraction of his total effort on task $A$ than his colleague and vice versa $j$ spends a higher fraction of his effort on task $B$. Let the equilibrium effort levels be $\hat{a}_{i A}, \hat{a}_{i B}, \hat{a}_{j A}, \hat{a}_{j B}$ and let $\hat{y}_{A}$ and $\hat{y}_{B}$ be the expected outcomes of respective tasks given these beliefs. Then $i$ is promoted when

$$
\begin{gathered}
\hat{a}_{j B}\left(y_{A}-E\left[\hat{y}_{A}\right]\right)-\hat{a}_{j A}\left(y_{B}-E\left[\hat{y}_{B}\right]\right)>\hat{a}_{i A}\left(y_{B}-E\left[\hat{y}_{B}\right]\right)-\hat{a}_{i B}\left(y_{A}-E\left[\hat{y}_{A}\right]\right) \\
\Leftrightarrow\left(\hat{a}_{i B}+\hat{a}_{j B}\right)\left(y_{A}-E\left[\hat{y}_{A}\right]\right)>\left(\hat{a}_{i A}+\hat{a}_{j A}\right)\left(y_{B}-E\left[\hat{y}_{B}\right]\right)
\end{gathered}
$$

It is important to note, that an agent can raise his probability of being promoted by increasing the outcome of the task, on which he is believed to 
exert a relatively higher fraction of his total effort. Conversely, he lowers the probability of promotion when he raises his effort level for the task on which he is believed to exert the lower fraction of the total effort. From these considerations it is straightforward to see that in any equilibrium $a_{i B}=0$ and $a_{j A}=0$ whenever $a_{i A} a_{j B}>a_{j A} a_{i B}$.

Agent $i$ is therefore promoted when

$$
\hat{a}_{j B}\left(y_{A}-E\left[\hat{y}_{A}\right]\right)>\hat{a}_{i A}\left(y_{B}-E\left[\hat{y}_{B}\right]\right) .
$$

Hence, agent $i$ 's equilibrium strategy on stage 1 is characterized by

$$
\max _{a_{i A}} \operatorname{Pr}\left(\hat{a}_{j B}\left(\theta_{i} a_{i A}-m \hat{a}_{i A}\right)>\hat{a}_{i A}\left(\theta_{j} \hat{a}_{j B}-m \hat{a}_{j B}\right)\right) B-c\left(a_{i A}\right)
$$

or

$$
\max _{a_{i A}} \operatorname{Pr}\left(\frac{a_{i A} \theta_{i}}{\hat{a}_{i A}}>\theta_{j}\right) B-c\left(a_{i A}\right) .
$$

By solving this optimization problem we can characterize pure strategy equilibria of the game: ${ }^{11}$

Proposition 7 In any pure strategy equilibrium in which both agents exert positive effort levels, each agent is held responsible and works only for one of the tasks and both work for different tasks. If agent 1 is responsible for task $A$ and agent 2 for task $B$, then the equilibrium effort levels are characterized by $a_{j A}^{*}=a_{i B}^{*}=0$ and

$$
\phi(0) \frac{m B}{\sqrt{2} \sigma}=a_{i A}^{*} c^{\prime}\left(a_{i A}^{*}\right)=a_{j B}^{*} c^{\prime}\left(a_{j B}^{*}\right) .
$$

Proof: See Appendix.

It is important to note, that there is no equilibrium in this model, in which both agents exert effort for the same task and therefore the responsibility for a single task will never be divided among two agents.

However, responsibility may well be divided for separate tasks. As we have seen, there may well be equilibria in which each agent is held responsible for a separate task. When the principal believes that agent $i$ works harder for task $A$ and agent $j$ works harder for task $B$ then, it is agent $i$ 's reputation that 
suffers, when the outcome of task $A$ is low and analogously $j^{\prime} s$ reputation when the same happens with the outcome of task $B$. Both agents then compete in a tournament and each of them has a strong incentive to work hard to lead his own task to a success.

Clearly, the equilibrium in which the two agents are responsible for separate tasks is opimal from a learning perspective. Only in this equilibrium the principal gets a signal on each agent's ability. Moreover, as the principal knows the equilibrium effort levels he can infer the abilities perfectly and will always promote the agent with the higher ability. This is not the case, if one agent is responsible for both tasks.

Proposition 7 has an additional important consequence: We can now get both agents to work and neither of them is idle when each is held responsible for a separate task. Indeed, we can show that for many cases the total effort exerted is larger when each agent is responisble for a task than when one agent is responsible for both:

Corollary 2 When $c^{\prime \prime \prime}(a) \geq 0$ then the total effort exerted on both tasks will always be larger when each agent is held responsible for a separate task than when one agent is responsible for both tasks.

Proof: See Appendix.

Finally, note that if the principal could endogenously determine the benefit of promotion $B$ for instance by adapting the wage structure of the organization she may even be able to implement first best effort levels. For instance, consider the case where the principal's revenue from the efforts on the two tasks is $\Pi=\pi\left(a_{i A}+a_{j A}\right)+\pi\left(a_{i B}+a_{j B}\right)$, where $\pi$ is some strictly concave function. Note that in any first best optimal solution both agents will then exert the same effort level and the efforts exerted for both tasks are the same. ${ }^{12}$ Furthermore, first best profits can then be attained when $a_{i A}=a_{j B}>0$ and $a_{j A}=a_{i B}=0$. Then, first best effort levels $a^{F B}=a_{i A}^{F B}=a_{j B}^{F B}$ are implemented by choosing $B$ such that (5) is met for $a^{F B}$. Similar to typical results in the tournament literature, it is straightforward that the principal 
will indeed optimally choose this value of $B$ when the agents have unlimited wealth.

Note that first-best effort levels can of course neither be attained in the no responsibility equilibrium nor when one agent is responsible for both tasks. Only, when each agent is held responsible for a separate task appropriate incentives are induced. This result is related to a result by Holmström and Milgrom (1991). They analyze a linear hidden action model in which two agents can work for several tasks and in which - as in our model — the efforts an agent spends on the tasks are perfectly substitutable in his cost function. They obtain the result that it is never optimal for the two agents to be rewarded for the outcome of the same task. Hence, as in our model the two agents optimally work for separate tasks. Note that in their model the allocation of tasks consists in the design of a formal incentive contract. Here the allocation of responsibility arises as an equilibrium outcome in a game where agents care for their career prospects.

\section{Conclusion}

We have provided a simple model to clarify the notion of responsibility in a multiagent career concerns framework. In the single task model we have shown that two types of equilibria may exist. Either no agent bears responsibility and, hence, no agent contributes effort to the task or there are sole responsibility equilibria in which exactly one agent contributes effort to the task and hence, the principal's beliefs on this agent's ability is adapted according to the outcome of the task.

The principal is of course always better off with a sole responsibility equilibrium, as then one agent has an incentive to exert effort on the task. This yields a simple formal explanation for the commonly felt principle that responsibility for a certain task should be allocated to one person as for instance stated in the phrase by Alexander Hamilton cited in beginning of the paper: "The sole and undivided responsibility of one man will naturally beget a livelier sense of duty and a more exact regard to reputation". ${ }^{13}$ 
We have then analyzed, which agent should be responsible for a given task if the agents differ in their abilities or the precision with which those abilities are known. Two aspects have been identified in our model, which are important for assigning responsibility. On the one hand, the principal has to consider which agent works harder, when being responsible for the task and which agent attains a higher expected output. On the other hand, she has to bear in mind that the assignment of responsibility generates information as she learns more about the ability of the responsible agent and this in turn is valuable as it helps to improve the promotion decision.

Finally, we have shown that first-best optimal incentives can be induced when there are several tasks. It is important to understand that efficiency can only be attained when holding the agents responsible for separate tasks. It is neither possible when no agent is responsible for a task, as then incentives are destroyed entirely, nor when one agent is responsible for both tasks. Hence, only a combination of an undivided responsibility for each task and the division of labor across tasks creates high powered incentives.

\section{Appendix}

\section{Calculation of expression (1):}

We know that for normally distributed random variables the following equation holds: ${ }^{14}$

$$
E[Y \mid X]=E[Y]+\frac{\operatorname{Cov}[X, Y]}{\operatorname{Var}[X]}(X-E[X]) .
$$

Applying this, we obtain

$$
\begin{aligned}
E\left[\theta_{i} \mid \theta_{i} a_{i}+\theta_{j} a_{j}\right] & =m_{i}+\frac{\operatorname{Cov}\left[\theta_{i}, \theta_{i} a_{i}+\theta_{j} a_{j}\right]}{\operatorname{Var}\left[\theta_{i} a_{i}+\theta_{j} a_{j}\right]}\left(\theta_{i} a_{i}+\theta_{j} a_{j}-E\left[\theta_{i} a_{i}+\theta_{j} a_{j}\right]\right) \\
& =m_{i}+\frac{a_{i} \sigma_{i}^{2}}{a_{i}^{2} \sigma_{i}^{2}+a_{j}^{2} \sigma_{j}^{2}}(y-E[y]) .
\end{aligned}
$$

\section{Proof of Lemma 1:}


The principal will promote agent $i$ iff $E\left[\theta_{i} \mid \theta_{i} a_{i}+\theta_{j} a_{j}\right]>E\left[\theta_{j} \mid \theta_{i} a_{i}+\theta_{j} a_{j}\right]$ or

$$
\begin{aligned}
m_{i}+ & \frac{a_{i} \sigma_{i}^{2}}{a_{i}^{2} \sigma_{i}^{2}+a_{j}^{2} \sigma_{j}^{2}}(y-E[y])>m_{j}+\frac{a_{j} \sigma_{j}^{2}}{a_{i}^{2} \sigma_{i}^{2}+a_{j}^{2} \sigma_{j}^{2}}(y-E[y]) \Leftrightarrow \\
& \frac{a_{i} \sigma_{i}^{2}-a_{j} \sigma_{j}^{2}}{a_{i}^{2} \sigma_{i}^{2}+a_{j}^{2} \sigma_{j}^{2}}(y-E[y])>m_{j}-m_{i} .
\end{aligned}
$$

If $a_{i} \sigma_{i}^{2}>a_{j} \sigma_{j}^{2}$ this is equivalent to

$$
y>E[y]-\frac{a_{i}^{2} \sigma_{i}^{2}+a_{j}^{2} \sigma_{j}^{2}}{a_{i} \sigma_{i}^{2}-a_{j} \sigma_{j}^{2}}\left(m_{i}-m_{j}\right) .
$$

If, however, $a_{i} \sigma_{i}^{2}=a_{j} \sigma_{j}^{2}$ this is equivalent to

$$
m_{i}>m_{j}
$$

\section{Proof of Proposition 1:}

Agent $i$ 's expected utility is determined by the probability of being promoted:

$$
\operatorname{Pr}\left(\theta_{i} a_{i} \geq \hat{a}_{i} m_{j}\right) B-c\left(a_{i}\right) .
$$

If $a_{i}=0$ and $\hat{a}_{i}>0$ this probability of being promoted is zero. The agent gets zero utility. If $a_{i}>0$, we can reformulate (6) and obtain:

$$
\max _{a_{i}} \operatorname{Pr}\left(\frac{\theta_{i}}{m_{j} \hat{a}_{i}} \geq \frac{1}{a_{i}}\right) B-c\left(a_{i}\right) .
$$

where

$$
\frac{\theta_{i}}{m_{j} \hat{a}_{i}} \sim N\left(\frac{m_{i}}{m_{j} \hat{a}_{i}}, \frac{\sigma_{i}^{2}}{\left(m_{j} \hat{a}_{i}\right)^{2}}\right) .
$$

Hence,

$$
\operatorname{Pr}\left(\frac{\theta_{i}}{m_{j} \hat{a}_{i}} \geq \frac{1}{a_{i}}\right)=1-\Phi\left(\frac{\frac{1}{a_{i}}-\frac{m_{i}}{m_{j} \hat{a}_{i}}}{\frac{\sigma_{i}}{m_{j} \hat{a}_{i}}}\right),
$$

and the maximization problem is given by:

$$
\max _{a_{i}} \Phi\left(\frac{1}{\sigma_{i}}\left(m_{i}-m_{j} \frac{\hat{a}_{i}}{a_{i}}\right)\right) B-c\left(a_{i}\right) .
$$


The first order condition is

$$
\phi\left(\frac{1}{\sigma_{i}}\left(m_{i}-m_{j} \frac{\hat{a}_{i}}{a_{i}}\right)\right) \frac{m_{j} \hat{a}_{i}}{\sigma_{i} a_{i}^{2}} B-c^{\prime}\left(a_{i}\right)=0 .
$$

Hence, if a pure strategy equilibrium with $a_{i}^{*}>0$ exists we must have that

$$
\phi\left(\frac{\Delta m}{\sigma_{i}}\right) \frac{m_{j}}{\sigma_{i}} B-a_{i}^{*} c^{\prime}\left(a_{i}^{*}\right)=0
$$

where $\Delta m=m_{j}-m_{i}$. This equation yields a unique strictly positive value for $a_{i}$ as a possible equilibrium strategy. Note that the first order condition is necessary but not sufficient as the objective function (8) will in general not be concave. Hence, we have to check that choosing $\hat{a}$ is indeed a global maximum for given beliefs $\hat{a}$. First, we compute the second derivative of the expected payoff from promotion, which is

$$
\left[\left(\frac{\hat{a}_{i}}{a_{i}} m_{j}-m_{i}\right) \frac{m_{j} \hat{a}_{i}}{\sigma_{i}^{2} a_{i}}-2\right] \frac{m_{j} \hat{a}_{i}}{\sigma_{i} a_{i}^{3}} \phi\left(\frac{m_{j} \hat{a}_{i}}{\sigma_{i} a_{i}}-\frac{m_{i}}{\sigma_{i}}\right) B .
$$

Note that there is a unique inflection point at:

$$
a_{I P}=\frac{m_{j} \hat{a}_{i}}{4 \sigma_{i}^{2}}\left(\sqrt{\left(m_{i}^{2}+8 \sigma_{i}^{2}\right)}-m_{i}\right) .
$$

The payoff from promotion is strictly convex below $a_{I P}$ and strictly concave above. At $\hat{a}_{i}$ the second derivative is negative iff $\left(m_{j}-m_{i}\right) \frac{m_{j}}{\sigma_{i}^{2}}-2<0$ which is always the case if $\sigma_{i}$ is sufficiently large. Hence, for large values of the variance, $\hat{a}_{i}>a_{I P}$ and $\hat{a}_{i}$ is the unique local maximum above $a_{I P}$.

If the global maximum would to be the left of $\hat{a}_{i}$ the agents payoff in such a global maximum is bounded from above by the gross payoff from promotion at the inflection point (as the winning probability is increasing in $a$ )

$$
\Phi\left(\frac{m_{i}}{\sigma_{i}}-\frac{4 \sigma_{i}}{\left(\sqrt{\left(m_{i}^{2}+8 \sigma_{i}^{2}\right)}-m_{i}\right)}\right) B
$$

A sufficient condition for the existence of an equilibrium is therefore that the agent's utility at $\hat{a}_{i}=a_{i}^{*}$ which is given by

$$
\Phi\left(\frac{m_{i}-m_{j}}{\sigma_{i}}\right) B-c\left(a_{i}^{*}\right)
$$


exceeds (10). Both functions are continuous in $\sigma_{i}$. Note from (9) that $a_{i}^{*}$ is strictly decreasing in $\sigma_{i}$ if $\sigma_{i}$ is large enough as ${ }^{15}$

$$
\frac{\partial\left(\frac{m_{j} B}{\sigma_{i}} \phi\left(\frac{\Delta m}{\sigma_{i}}\right)\right)}{\partial \sigma_{i}}<0 \Leftrightarrow \sigma_{i}^{2}>\Delta m^{2} .
$$

and it converges to zero for $\sigma_{i} \rightarrow \infty$. Hence, expression (11) tends to $\frac{B}{2}$ as $\sigma_{i}$ approaches infinity. But the limit of the upper boundary (10) can be computed as

$$
\lim _{\sigma_{i} \rightarrow \infty} \Phi\left(\frac{m_{i}}{\sigma_{i}}-\frac{4 \sigma_{i}}{\left(\sqrt{\left(m_{i}^{2}+8 \sigma_{i}^{2}\right)}-m_{i}\right)}\right) B=\Phi(-\sqrt{2}) B
$$

which is smaller than $\frac{B}{2}$. Hence, for sufficiently large $\sigma_{i}$ the unique global maximizer is $\hat{a}_{i}$ and the equilibrium exists.

\section{Proof of Proposition 2:}

If $\sigma_{i}^{2} a_{i}=\sigma_{j}^{2} a_{j}$ and $m_{i}=m_{j}$ the principal is indifferent on whom to promote. She can choose an arbitrary promotion strategy $p_{i}(y)$ where this denotes the probability of promoting agent $i$ (and therefore $p_{j}(y)=1-p_{i}(y)$ ). Suppose that there is an equilibrium with $a_{i}>0$ and $a_{j}>0$ in this case. First, we show that for any equilibrium there will be two separate unilateral deviations by the two agents which lead to the same outcome distribution. Then we show that it can never be the case that both deviations are unprofitable.

Note that

$$
\begin{aligned}
\left(a_{i}^{\prime} \theta_{i}+a_{j} \theta_{j}\right) & \sim N\left(\left(a_{i}^{\prime}+a_{j}\right) m,\left(a_{i}^{\prime 2} \sigma_{i}^{2}+a_{j}^{2} \sigma_{i}^{2}\right)\right) \text { and } \\
\left(a_{i} \theta_{i}+a_{j}^{\prime} \theta_{j}\right) & \sim N\left(\left(a_{i}+a_{j}^{\prime}\right) m,\left(a_{i}^{2} \sigma_{i}^{2}+a_{j}^{\prime 2} \sigma_{i}^{2}\right)\right) .
\end{aligned}
$$

Two unilateral deviations $a_{i}^{\prime}$ and $a_{j}^{\prime}$ lead to the same outcome distribution when

$$
a_{i}^{\prime}+a_{j}=a_{i}+a_{j}^{\prime} \quad \text { and } \quad\left(a_{i}^{\prime 2} \sigma_{i}^{2}+a_{j}^{2} \sigma_{j}^{2}\right)=\left(a_{i}^{2} \sigma_{i}^{2}+a_{j}^{\prime 2} \sigma_{j}^{2}\right)
$$

We have to distinguish two cases: 
(i) If $\sigma_{i}^{2}=\sigma_{j}^{2}$ we must have that $a_{i}=a_{j}$ as $\sigma_{i}^{2} a_{i}=\sigma_{j}^{2} a_{j}$. Then the deviations $a_{i}^{\prime}=0$ and $a_{j}^{\prime}=0$ satisfy conditions (12).

(ii) If $\sigma_{i}^{2} \neq \sigma_{j}^{2}$ it is straightforward to check that

$$
a_{i}^{\prime}=\frac{\left(a_{i}\left(\sigma_{i}^{2}+\sigma_{j}^{2}\right)-2 a_{j} \sigma_{j}^{2}\right)}{\sigma_{j}^{2}-\sigma_{i}^{2}} \text { and } a_{j}^{\prime}=\frac{\left(a_{j}\left(\sigma_{i}^{2}+\sigma_{j}^{2}\right)-2 a_{i} \sigma_{i}^{2}\right)}{\sigma_{i}^{2}-\sigma_{j}^{2}}
$$

is a solution to the system of equations defined by conditions (12). Furthermore, $a_{i}^{\prime}$ and $a_{j}^{\prime}$ are positive when $\sigma_{i}^{2} a_{i}=\sigma_{j}^{2} a_{j}$. Hence, for any equilibrium there exist separate feasible unilateral deviations by each of the agents leading both to the same outcome distribution.

But if $a_{i}$ and $a_{j}$ are chosen in equilibrium, we must have that

$$
\begin{aligned}
& B \cdot E\left[p_{i}\left(a_{i} \theta_{i}+a_{j} \theta_{j}\right)\right]-c\left(a_{i}\right) \geq B \cdot E\left[p_{i}\left(a_{i}^{\prime} \theta_{i}+a_{j} \theta_{j}\right)\right]-c\left(a_{i}^{\prime}\right) \text { and } \\
& B \cdot E\left[p_{j}\left(a_{i} \theta_{i}+a_{j} \theta_{j}\right)\right]-c\left(a_{j}\right) \geq B \cdot E\left[p_{j}\left(a_{i} \theta_{i}+a_{j}^{\prime} \theta_{j}\right)\right]-c\left(a_{j}^{\prime}\right)
\end{aligned}
$$

which are equivalent to

$$
\begin{aligned}
B \cdot\left(E\left[p_{i}\left(a_{i} \theta_{i}+a_{j} \theta_{j}\right)\right]-E\left[p_{i}\left(a_{i}^{\prime} \theta_{i}+a_{j} \theta_{j}\right)\right]\right) & \geq c\left(a_{i}\right)-c\left(a_{i}^{\prime}\right) \text { and }(13) \\
B \cdot\left(E\left[p_{i}\left(a_{i} \theta_{i}+a_{j} \theta_{j}\right)\right]-E\left[p_{i}\left(a_{i} \theta_{i}+a_{j}^{\prime} \theta_{j}\right)\right]\right) & \leq c\left(a_{j}^{\prime}\right)-c\left(a_{j}\right) .
\end{aligned}
$$

But as $E\left[p_{i}\left(a_{i}^{\prime} \theta_{i}+a_{j} \theta_{j}\right)\right]=E\left[p_{i}\left(a_{i} \theta_{i}+a_{j}^{\prime} \theta_{j}\right)\right]$, the left hand sides of equations (13) and (14) are identical. This implies that $c\left(a_{j}^{\prime}\right)-c\left(a_{j}\right) \geq c\left(a_{i}\right)-$ $c\left(a_{i}^{\prime}\right)$ and, hence, when $a_{i}^{\prime} \leq a_{i}$ we must have $a_{j}^{\prime} \geq a_{j}$. But the first condition in (12) is equivalent to $a_{i}^{\prime}-a_{i}=a_{j}^{\prime}-a_{j}$ which leads to a contradiction.

\section{Proof of Proposition 3:}

The expected profit if agent $i(j)$ is held responsible is $a_{i}^{*} m_{i}\left(a_{j}^{*} m_{j}\right)$. We know that for $\sigma_{i}=\sigma_{j}=\sigma$,

$$
\begin{aligned}
& \frac{m_{j} B}{\sigma} \phi\left(\frac{\Delta m}{\sigma}\right)-a_{i}^{*} c^{\prime}\left(a_{i}^{*}\right)=0, \\
& \frac{m_{i} B}{\sigma} \phi\left(\frac{\Delta m}{\sigma}\right)-a_{j}^{*} c^{\prime}\left(a_{j}^{*}\right)=0,
\end{aligned}
$$


which are equivalent to

$$
\begin{aligned}
a_{i}^{*} m_{i} & =\frac{\frac{m_{j} B}{\sigma} \phi\left(\frac{\Delta m}{\sigma}\right)}{c^{\prime}\left(a_{i}^{*}\right)} m_{i} \\
a_{j}^{*} m_{j} & =\frac{\frac{m_{i} B}{\sigma} \phi\left(\frac{\Delta m}{\sigma}\right)}{c^{\prime}\left(a_{j}^{*}\right)} m_{j}
\end{aligned}
$$

We get that $a_{i}^{*} m_{i}>a_{j}^{*} m_{j}$ iff

$$
\begin{aligned}
\frac{\frac{m_{j} B}{\sigma} \phi\left(\frac{\Delta m}{\sigma}\right)}{c^{\prime}\left(a_{i}^{*}\right)} m_{i} & >\frac{\frac{m_{i} B}{\sigma} \phi\left(\frac{\Delta m}{\sigma}\right)}{c^{\prime}\left(a_{j}^{*}\right)} m_{j} \Leftrightarrow \\
c^{\prime}\left(a_{j}^{*}\right) & >c^{\prime}\left(a_{i}^{*}\right) \Leftrightarrow a_{j}^{*}>a_{i}^{*} .
\end{aligned}
$$

As we have seen this is the case iff $m_{i}>m_{j}$.

\section{Proof of Proposition 5:}

By adapting a result from Gourieroux and Monfort (1989) (p. 529), we first get that the expected value of the maximum of normally distributed random variables is given by

$E[\max \{X, Y\}]=m_{X}+\sqrt{\sigma_{X}^{2}+\sigma_{Y}^{2}} \phi\left(\frac{m_{X}-m_{Y}}{\sqrt{\sigma_{X}^{2}+\sigma_{Y}^{2}}}\right)-\left(m_{X}-m_{Y}\right) \Phi\left(\frac{m_{Y}-m_{X}}{\sqrt{\sigma_{X}^{2}+\sigma_{Y}^{2}}}\right)$.

Let again $\Delta m=m_{i}-m_{j}$. We obtain

$$
E\left[\max \left\{\theta_{i}, m_{j}\right\}\right]=m_{i}+\sqrt{\sigma_{i}^{2}} \phi\left(\frac{\Delta m}{\sigma_{i}}\right)-\Delta m \Phi\left(\frac{-\Delta m}{\sigma_{i}}\right) .
$$

The principal prefers to promote agent $i$ if

$$
\begin{aligned}
\Delta m+\sigma_{i} \phi\left(\frac{\Delta m}{\sigma_{i}}\right)-\Delta m \Phi\left(-\frac{\Delta m}{\sigma_{i}}\right) & \geq \sigma_{j} \phi\left(\frac{\Delta m}{\sigma_{j}}\right)+\Delta m \Phi\left(\frac{\Delta m}{\sigma_{j}}\right) \Leftrightarrow \\
\Delta m+\sigma_{i} \phi\left(\frac{\Delta m}{\sigma_{i}}\right)-\Delta m\left(1-\Phi\left(\frac{\Delta m}{\sigma_{i}}\right)\right) & \geq \sigma_{j} \phi\left(\frac{\Delta m}{\sigma_{j}}\right)+\Delta m \Phi\left(\frac{\Delta m}{\sigma_{j}}\right) \Leftrightarrow \\
\sigma_{i} \phi\left(\frac{\Delta m}{\sigma_{i}}\right)+\Delta m \Phi\left(\frac{\Delta m}{\sigma_{i}}\right) & \geq \sigma_{j} \phi\left(\frac{\Delta m}{\sigma_{j}}\right)+\Delta m \Phi\left(\frac{\Delta m}{\sigma_{j}}\right) .
\end{aligned}
$$

Note that for $\sigma_{i}=\sigma_{j}$ both sides are equal and the principal is indifferent whom to promote whatever the value of $\Delta m$. To see that she prefers to 
promote agent $i$ iff $\sigma_{i} \geq \sigma_{j}$ check that $\sigma \phi\left(\frac{\Delta m}{\sigma}\right)+\Delta m \Phi\left(\frac{\Delta m}{\sigma}\right)$ is strictly increasing in $\sigma$ whatever the sign of $\Delta m$ :

$$
\frac{\partial}{\partial \sigma}\left(\sigma \phi\left(\frac{\Delta m}{\sigma}\right)+\Delta m \Phi\left(\frac{\Delta m}{\sigma}\right)\right)=\phi\left(\frac{\Delta m}{\sigma}\right)-\frac{\Delta m}{\sigma} \phi^{\prime}\left(\frac{\Delta m}{\sigma}\right)-\frac{\Delta m^{2}}{\sigma^{2}} \phi\left(\frac{\Delta m}{\sigma}\right) .
$$

Using that $\phi^{\prime}(x)=-x \phi(x)$ this expression is simply equal to $\phi\left(\frac{\Delta m}{\sigma}\right)>0$.

\section{Proof of Proposition 6:}

As we have seen, when only $a_{i A}>0$ we can proceeed as in the proof of Proposition 1 and obtain 4 . When $a_{i A}, a_{i B}>0$ we must have that $y_{A}=\frac{\hat{a}_{i A}}{\hat{a}_{i B}} y_{B}$ as $y_{A}=\hat{a}_{i A} \theta_{i}$ and $y_{B}=\hat{a}_{i B} \theta_{i}$ on the equilibrium path .We specify the out-ofequilibrium beliefs such that the principal will always believe that the other agent $j$ has a higher ability whenever this is not the case. Hence, if there is an internal solution to his optimization problem the agent will always choose $a_{i B}$ such that $a_{i A}=\frac{\hat{a}_{i A}}{\hat{a}_{i B}} a_{i B}$. The total effort exerted is therefore $a_{i A}+a_{i B}=a_{i A}\left(1+\frac{\hat{a}_{i B}}{\hat{a}_{i A}}\right)$. Agent $i$ will be promoted when $a_{i A} \theta_{i}>\hat{a}_{i A} m$ and his objective function therefore is

$$
\operatorname{Pr}\left(\theta_{i} a_{i A} \geq \hat{a}_{i A} m\right) B-c\left(a_{i A}\left(1+\frac{\hat{a}_{i B}}{\hat{a}_{i A}}\right)\right) .
$$

If $a_{i}=0$ and $\hat{a}_{i}>0$ this probability of being promoted is zero and so is his utility. If $a_{i}>0$, we can reformulate (15) and obtain:

$$
\max _{a_{i}} \operatorname{Pr}\left(\frac{\theta_{i}}{m \hat{a}_{i A}} \geq \frac{1}{a_{i A}}\right) B-c\left(a_{i A}\left(1+\frac{\hat{a}_{i B}}{\hat{a}_{i A}}\right)\right) .
$$

where

$$
\frac{\theta_{i}}{m \hat{a}_{i A}} \sim N\left(\frac{1}{\hat{a}_{i A}}, \frac{\sigma^{2}}{\left(m \hat{a}_{i A}\right)^{2}}\right) .
$$

Hence,

$$
\begin{aligned}
\operatorname{Pr}\left(\frac{\theta_{i}}{m \hat{a}_{i A}} \geq \frac{1}{a_{i A}}\right) & =1-\Phi\left(\frac{\frac{1}{a_{i A}}-\frac{1}{\hat{a}_{i A}}}{\frac{\sigma_{i}}{m \hat{a}_{i A}}}\right) \\
& =1-\Phi\left(\left(\frac{\hat{a}_{i A}}{a_{i A}}-1\right) \frac{m}{\sigma_{i}}\right)=\Phi\left(\left(1-\frac{\hat{a}_{i A}}{a_{i A}}\right) \frac{m}{\sigma_{i}}\right)
\end{aligned}
$$

and the maximization problem is given by:

$$
\max _{a_{i}} \Phi\left(\frac{m}{\sigma_{i}}\left(1-\frac{\hat{a}_{i A}}{a_{i A}}\right)\right) B-c\left(a_{i A}\left(1+\frac{\hat{a}_{i B}}{\hat{a}_{i A}}\right)\right) .
$$


The first order condition is

$$
\phi\left(\frac{m}{\sigma_{i}}\left(1-\frac{\hat{a}_{i A}}{a_{i A}}\right)\right) \frac{m \hat{a}_{i A}}{\sigma_{i} a_{i A}^{2}} B-c^{\prime}\left(a_{i A}\left(1+\frac{\hat{a}_{i B}}{\hat{a}_{i A}}\right)\right)\left(1+\frac{\hat{a}_{i B}}{\hat{a}_{i A}}\right)=0 .
$$

Hence, in a pure strategy equilibrium with $a_{i A}^{*}>0$ we must have that

$$
\begin{gathered}
\phi(0) \frac{m}{\sigma_{i} a_{i A}} B-c^{\prime}\left(a_{i A}+a_{i B}\right)\left(1+\frac{a_{i B}}{a_{i A}}\right)=0 . \\
\phi(0) \frac{m}{\sigma_{i}} B-c^{\prime}\left(a_{i A}+a_{i B}\right)\left(a_{i A}+a_{i B}\right)=0 .
\end{gathered}
$$

Note that this equation uniquely defines the total effort exerted in any equilibrium $a=a_{i A}+a_{i B}$. However, there is a continuum of such equilibria. To see that such equilibria exist whenever $\sigma$ is sufficiently large we can proceed as in the proof of Proposition 1 by treating this as a single variable optimization problem with the strictly convex cost function $c^{N}\left(a_{i A}\right)=c\left(a_{i A}\left(1+\frac{\hat{a}_{i B}}{\hat{a}_{i A}}\right)\right)$.

\section{Proof of Proposition 7:}

Agent $i$ maximizes

$$
\max _{a_{i A}} \operatorname{Pr}\left(\theta_{j}-\frac{a_{i A} \theta_{i}}{\hat{a}_{i A}}<0\right) B-c\left(a_{i A}\right) .
$$

Let $\zeta=\theta_{j}-\frac{a_{i A} \theta_{i}}{\hat{a}_{i A}}$. Hence, $E[\zeta]=m-\frac{a_{i A} m}{\hat{a}_{i A}}=\left(1-\frac{a_{i A}}{\hat{a}_{i A}}\right) m$ and $\operatorname{Var}[\zeta]=$ $\sigma^{2}+\left(\frac{a_{i A}}{\hat{a}_{i A}}\right)^{2} \sigma^{2}=\sigma^{2}\left(1+\frac{a_{i A}^{2}}{\hat{a}_{i A}^{2}}\right)$. Hence,

$$
\operatorname{Pr}(\zeta<0)=\Phi\left(-\frac{\left(1-\frac{a_{i A}}{\hat{a}_{i A}}\right) m}{\sigma \sqrt{\frac{\hat{a}_{i A}^{2}+a_{i A}^{2}}{\hat{a}_{i A}^{2}}}}\right)=\Phi\left(-\frac{m\left(\hat{a}_{i A}-a_{i A}\right)}{\sigma \sqrt{\hat{a}_{i A}^{2}+a_{i A}^{2}}}\right)
$$

and the optimization problem is equivalent to

$$
\max _{a_{i A}} \Phi\left(-\frac{m\left(\hat{a}_{i A}-a_{i A}\right)}{\sigma \sqrt{\hat{a}_{i A}^{2}+a_{i A}^{2}}}\right) B-c\left(a_{i A}\right) .
$$

The first order condition is

$$
\phi\left(-\frac{m\left(\hat{a}_{i A}-a_{i A}\right)}{\sigma \sqrt{\hat{a}_{i A}^{2}+a_{i A}^{2}}}\right) \frac{m}{\sigma}\left(\frac{1}{\sqrt{\hat{a}_{i A}^{2}+a_{i A}^{2}}}+\frac{a_{i A}\left(\hat{a}_{i A}-a_{i A}\right)}{\left(\hat{a}_{i A}^{2}+a_{i A}^{2}\right)^{\frac{3}{2}}}\right) B-c^{\prime}\left(a_{i A}\right)=0
$$


In equilibrium we must have that $\hat{a}_{i A}=a_{i A}$ and therefore

$$
\phi(0) \frac{m B}{\sqrt{2} \sigma}-a_{i A} c^{\prime}\left(a_{i A}\right)=0 .
$$

\section{Proof of Corollary 2:}

Let $\psi(a)=a \cdot c^{\prime}(a)$. When $c^{\prime \prime \prime}(a) \geq 0$ then $\psi(a)$ is strictly convex as $\psi^{\prime \prime}(a)=$ $2 c^{\prime \prime}(a)+a c^{\prime \prime \prime}(a)>0$. Hence, $\psi^{-1}(x)$ is strictly concave and monotonically increasing. Let the total effort when only one agent is responsible for both tasks be $a_{O}^{*}$ and when the two agents are responsible each for one of the tasks be $a_{T}^{*}$. Then from Propositions 6 and 7 we get that

$$
a_{O}^{*}=\psi^{-1}\left(\phi(0) \frac{m B}{\sigma}\right) \text { and } a_{T}^{*}=2 \psi^{-1}\left(\phi(0) \frac{m B}{\sqrt{2} \sigma}\right) \text {. }
$$

But then we have that

$$
a_{T}^{*}=2 \psi^{-1}\left(\phi(0) \frac{m B}{\sqrt{2} \sigma}\right)>2 \psi^{-1}\left(\frac{1}{2} \phi(0) \frac{m B}{\sigma}\right)>\psi^{-1}\left(\phi(0) \frac{m B}{\sigma}\right)=a_{O}^{*},
$$

where the first inequality follows from the monotonicity and the second from the strict concavity of $\psi^{-1}(x)$.

\section{References}

Aghion, P. and Tirole, J. (1997): Formal and Real Authority in Organizations. Journal of Political Economy, 105, pp. 1-29.

Baker, G., Gibbons, R. and Murphy, K. J. (1999): Informal Authority in Organizations. Journal of Law, Economics and Organization, 15, pp. 5673.

Bhattacharya, S. and Guasch, J. L. (1988): Heterogeneity, Tournaments, and Hierarchies. Journal of Political Economy, 96, pp. 867-881. 
Dewatripont, M., Jewitt, I. and Tirole, J. (1999a): The Economics of Career Concerns, Part I: Comparing Information Structures. Review of Economic Studies, 66, pp. 183-198.

Dewatripont, M., Jewitt, I. and Tirole, J. (1999b): The Economics of Career Concerns, Part II: Application to Missions and Accountability of Government Agencies. Review of Economic Studies, 66, pp. 199-207.

Fairburn, J. and Malcomson, J. (1994): Rewarding Performance by Promotion to a Different Job. European Economic Review, 38, pp. 683-690.

Fairburn, J. and Malcomson, J. (2001): Performance, Promotion, and the Peter Principle. Review of Economic Studies, 68, pp. 45-66.

Farrell, J. and Rabin, M. (1996): Cheap Talk. Journal of Economic Perspectives, 10, pp. 103-118.

Gourieroux, C. and Monfort, A. (1989): Statistique et Modèles Économétriques, Vol. 2. Economica, Paris.

Green, J. R. and Stokey, N. L. (1983): A Comparison of Tournaments and Contracts. Journal of Political Economy, 91, pp. 349-364.

Hamilton, A., Madison, J. and Jay, J. (1961): The Federalist Papers. Penguin, New York.

Holmström, B. and Milgrom, P. (1991): Multitask Principal-Agent Analyses: Incentive Contracts, Asset Ownership and Job Design. Journal of Law, Economics and Organization, 7, pp. 24-52.

Holmström, B. (1982): Managerial Incentive Problems - A Dynamic Perspective. In: Essays in Economics and Management in Honor of Lars Wahlbeck, Helsinki.

Lazear, E. P. (1989): Pay Equality and Industrial Politics. Journal of Political Economy, 97, pp. 561-80. 
Lazear, E. P. and Rosen, S. (1981): Rank-Order Tournaments as Optimum Labor Contracts. Journal of Political Economy, 89, pp. 841-864.

Manove, M. (1997): Job Responsibility, Pay, and Promotion. The Economic Journal, 107, pp. 85-103.

Meyer, M. (1994): The Dynamics of Learning with Team Production: Implications for Task Assignment. Quarterly Journal of Economics, 109, pp. $1157-1184$.

Nalebuff, B. J. and Stiglitz, J. E. (1983): Prizes and Incentives: Towards a General Theory of Compensation and Competition. Bell Journal of Economics, 3, pp. 21-43.

Prendergast, C. J. (1993): The Role of Promotion in Inducing Specific Human Capital Acquisition. Quarterly Journal of Economics, 108, pp. 523-34.

Prendergast, C. J. (1995): A Theory of Responsibility in Organizations. Journal of Labor Economics, 13, pp. 387-400.

Sliwka, D. (2001): On the Costs and Benefits of Delegation in Organizations. Journal of Institutional and Theoretical Economics, 157, pp. 568-590.

Waldman, M. (1984): Job Assignments, Signalling, and Efficiency. Rand Journal of Economics, 15, pp. 255-267.

Waldman, M. (1990): Up-or-Out Contracts: A Signaling Perspective. Journal of Labor Economics, 8, pp. 230-50.

Zabojník, J. and Bernhardt, D. (2001): Corporate Tournaments, Human Capital Acquisition, and the Firm Size Wage Relation. Review of Economic Studies, 68, pp. 693-716. 


\section{Notes}

${ }^{1}$ Dewatripont et al. (1999a) provide more general results for a singleagent career concerns model for instance by dropping specific assumptions on distribution functions.

${ }^{2}$ For the relationship between informal and formal authority when a principal can allocate formal decision rights compare Aghion and Tirole (1997). Baker et al. (1999) show that informal commitment may be feasible and beneficial if an infinitely repeated game is considered.

${ }^{3}$ In the latter respect the paper is related to Meyer (1994) who studies how the necessity of learning agents' abilities affects the task assignment policy of a firm in a team production framework without incentive problems.

${ }^{4}$ Unlike in Holmström (1982) and Dewatripont et al. (1999b) in these papers the market cannot observe the exact output but only whether the agent has been promoted.

${ }^{5}$ The computation of this expression is shown in the appendix.

${ }^{6}$ Here, $\phi($.$) denotes the density of a standard normal distribution.$

${ }^{7}$ Note that a similar assumption typically is required for the existence of a pure strategy equilibrium in most tournament models (compare for instance Lazear and Rosen (1981) p.845 or Bhattacharya and Guasch (1988) p.871).

${ }^{8}$ Sliwka (2001) shows that a principal will sometimes delegate a decision to an agent with imperfectly known ability instead of making it herself even if the agent has a lower expected ability as it may turn out that the agent is more talented.

${ }^{9}$ Note that on the quilibrium path the principal must always observe $y_{B}=$ 0 . By specifing that the principal's prior beliefs are unaffected for any off-the equilibrium path observation $y_{B} \neq 0$ such an equilibrium can be sustained 
as will of course make no sense to exert any effort on task $B$.

${ }^{10}$ Compare for instance Gourieroux and Monfort (1989) p. 524 for the proof that for two vectors of normally distributed random variables $X, Y$ the following holds: $E[Y \mid X]=E[Y]+\operatorname{Cov}[X, Y](\operatorname{Var}[X])^{-1}(X-E[X])$.

${ }^{11}$ Unfortunately, I am unable to characterize proper analytical conditions for the existence of the equilibrium. However, numerical simulations show that - as in the previous results - it exists for large values of $\sigma$. For instance, if $B=1, m=1$ and $c=1$ the equilibrium exists if $\sigma=1$ but not if $\sigma=0.1$.

${ }^{12}$ If both agents exerted different effort levels the total costs could be reduced due to the convexity of $c(a)$ by lowering the effort of the hard working agent for one task and increasing the effort level of the other agent by the same amount.

By a similar argument: If the total effort exerted on task $A$ would be higher than that on task $B$ then efficiency could be raised due to the concavity of $\pi(a)$ by increasing the effort exerted by one of the agents on $B$ and decreasing that on $A$ by the same amount.

${ }^{13}$ Compare The Federalist Papers No. 76 in Hamilton et al. (1961), p. 455.

${ }^{14}$ Compare for instance Gourieroux and Monfort (1989) p. 524.

${ }^{15}$ Note that $\phi^{\prime}(x)=-x \phi(x)$ for the density of the standard normal distribution. 